\title{
The Gated X-ray Detector for the National Ignition Facility
}

J. A. Oertel, C. Barnes, T. Archuleta, L. Casper, V. Fatherley, T. Heinrichs, R. King, D. Landers, F. Lopez, P. Sanchez, G. Sandoval, L. Schrank, P. Walsh, P. Bell, M. Brown, R. Costa, J. Holder, S. Montalongo, N. Pederson

September 6, 2006 
This document was prepared as an account of work sponsored by an agency of the United States Government. Neither the United States Government nor the University of California nor any of their employees, makes any warranty, express or implied, or assumes any legal liability or responsibility for the accuracy, completeness, or usefulness of any information, apparatus, product, or process disclosed, or represents that its use would not infringe privately owned rights. Reference herein to any specific commercial product, process, or service by trade name, trademark, manufacturer, or otherwise, does not necessarily constitute or imply its endorsement, recommendation, or favoring by the United States Government or the University of California. The views and opinions of authors expressed herein do not necessarily state or reflect those of the United States Government or the University of California, and shall not be used for advertising or product endorsement purposes.

This work was performed under the auspices of the U.S. Department of Energy by University of California, Lawrence Livermore National Laboratory under Contract W-7405-Eng-48. 


\title{
The Gated X-ray Detector for the National Ignition Facility
}

\author{
John A. Oertel, Cris Barnes, Tom Archuleta, Larry Casper, Valerie Fatherley, Todd \\ Heinrichs, Robert King, Doug Landers, Frank Lopez, Phillip Sanchez, George Sandoval, \\ Lou Schrank, Peter Walsh \\ Los Alamos National Laboratory, Los Alamos New Mexico 87544
}

Perry Bell, Matt Brown, Robert Costa, Joe Holder, Sam Montalongo

Lawrence Livermore National Laboratory, Livermore, California 94551-0808

\author{
Neal Pederson \\ VI Control Systems Ltd, Los Alamos, NM 87544
}

\begin{abstract}
Two new gated x-ray imaging cameras have recently been designed, constructed and delivered to the National Ignition Facility in Livermore, CA. These Gated X-ray Detectors are each designed to fit within an aluminum airbox with a large capacity cooling plane and are fitted with an array of environmental housekeeping sensors. These instruments are significant different from earlier generations of gated x-ray images due in parts to an innovative impendence matching scheme, advanced phosphor screens, pulsed phosphor circuits, precision assembly fixturing, unique system monitoring and complete remote computer control. Preliminary characterization has shown repeatable uniformity between imaging strips, improved spatial resolution and no detectable impendence reflections.
\end{abstract}

\section{INTRODUCTION}

Gated x-ray imaging cameras commonly used by the international Inertial Confinement Fusion (ICF) and High Energy Density Physics (HEDP) community are time-dependent, two-dimensional imagers ${ }^{1}$. These instruments are important to researchers who require many sequential image frames, with adjustable temporal resolutions from $40 \mathrm{ps}$ to several nanoseconds, spatial resolution of $5 \mu \mathrm{m}$ (image magnified), spectral sensitivity from $0.2-10 \mathrm{keV}$, several orders of magnitude dynamic range, and centimeter scale image size. Although fundamentally similar to the original cameras developed over 20 years ago, the individual components that make up the latest generation of instruments have progressively improved. Primarily used at large laser facilities and required to operate in vacuum, these cameras are either mounted on a vacuum flange or loaded into an insertable mechanism and translated inside a vacuum target chamber.

Recently, Lawrence Livermore National Laboratory (LLNL) and the National Ignition Facility (NIF) commissioned Los Alamos National Laboratory (LANL) to construct the next generation gated $\mathrm{x}$-ray imager for the NIF laser. Named the Gated X-ray Detector (GXD), this new instrument takes advantage of all the technology developed to date to include improved electrical impedance transformation, pulsed phosphor high-voltage, remote computer control, advanced phosphor screens and custom CCD coupled cameras. See figure 1. Originally, the scope was to construct 4 identical instruments, but due to funding restraints the number was reduced to 2 instruments.

One important mission of the GXD is to measure laser performance parameters such as laser beam quality, profile and pointing all as a function of time. Additionally, the instrument is intended to acquire 2D spatially or 1D spectrally time resolved images of target features. Some specific examples are: spectral emission from targets ${ }^{2}$, spatially resolved foil trajectories $^{3}$, hydrodynamic instability growth information ${ }^{4}$, shock front propagation ${ }^{5}$ and $x$-ray time history for ignition physics ${ }^{6}$.

The GXD specifications include spectral sensitivities from $200 \mathrm{~nm}$ to $17.5 \mathrm{keV}$, photocathode active area of $30 \mathrm{~mm}$ square, four $10 \mathrm{ohm} 7.5 \mathrm{~mm}$ striplines, temporal window adjustable from 70 ps to $1500 \mathrm{ps}$, independent gain for each stripline, spatial resolution of $50 \mu \mathrm{m}$, independent strip delays up to 44 ns and pulsed phosphor.

The environment the GXD is designed to operate is harsh compared to earlier generations of instruments. This is one of the primary factors the GXD was designed to fit within an aluminum airbox allowing protection of the sensitive electronics.

The NIF chamber environment the GXD was designed to operate is: neutron flux of $10^{13}-1.5 \mathrm{~m}$ from the center of the target chamber, an unconverted laser light up to $100 \mathrm{~kJ}$ of $1.06 \mathrm{um}$, a vacuum of less-than $4 \mathrm{x}$ $10^{-5}$ Torr, and a magnetic field of 1 Gauss. 
Additionally, the instrument was required to adhere to the strict NIF cleanliness and material compatibility specifications and guidelines.
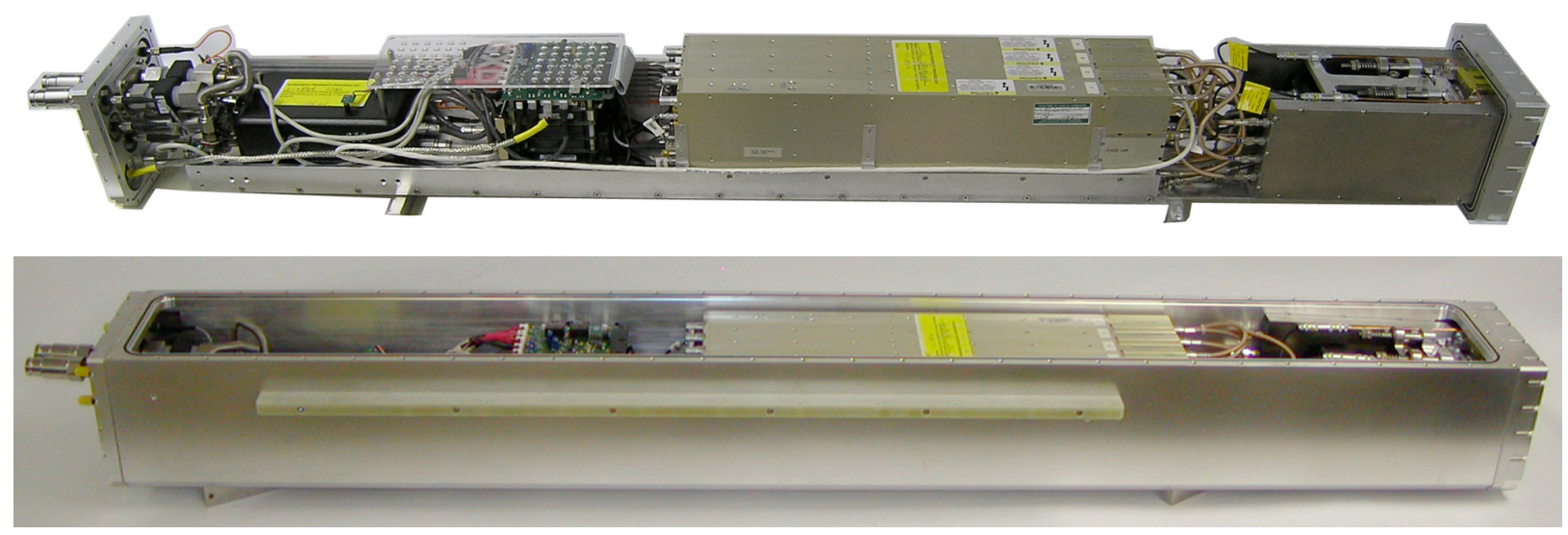

FIG. 1. The Gated X-ray Detector pulled outside of the air box (top) and within (bottom). Various types of imaging and spectrometer nosecones can be placed in front of the detector when the instrument is in the NIF vacuum chamber.

This remainder of this paper will describe the electrical and mechanical details of the instrument and some example performance characterization results.

\section{GATED X-RAY DETECTOR ANATOMY AND CONCEPT OF OPERATIONS}

The GXD is made up of 5 basic electrical and mechanical components: 1) The airbox 2) cooling plane mounting structure, 3) MCP module and CCD camera system, 4) Gating electronics package and 5) PC104 onboard computer plus a housekeeping sensor package. See figure 2. The instrument was designed to interface to the NIF's target chamber by way of a diagnostic instrument manipulator (DIM) ${ }^{7}$ and also to be backward compatible with the smaller Omega laser facility's TIM. ${ }^{8}$ Both of these mechanisms allow the instrument to be put under vacuum and translated into position inside the target chamber where it is precision aligned and readied for acquisition tasks. A cable bundle containing cooling water lines, 28 VDC power, fiberoptic triggers (pulser, CCD and phosphor), monitor, and ethernet lead from the instrument airbox through vacuum feedthroughs in the DIM and out to the NIF facility infrastructure. Instrument operations are intended to be compatible with the NIF, two-hour, shot cycle allowing for remote instrument monitoring and adjustment of sensitivity, timing, voltages, current and health.

Once a user decides on a spectrometer or imaging nosecone to be placed in front of the detector ${ }^{9}$ and the instrument is configured it is translated into the target chamber. Communications with the GXD are established using the TCP/IP ethernet based Diagnostic Computer (DC). The DC is local in the target bay and acts as the liaison between the front-end processor (FEP) and the GXD camera. Following confirmation of GXD functionality and health the GXD begins listening to the NIF integrated timing system (ITS) for the shot countdown or NIF shot life cycle and a CCD background image is acquired and stored remotely. At shot time, optical triggers are sent to the GXD pulser, phosphor and CD camera and an analog trigger monitor is sent out to a digitizing oscilloscope located in the shielded NIF mezzanine. The CCD camera is then read out and stored remotely with all the camera settings in HDF file format of $\sim 40 \mathrm{Mb}$ per shot. Concurrently the GXD system health (temperature, pressure, vacuum, voltage and current) is periodically polled, logged and displayed in the diagnostic control room station. If a critical parameter shows out of range, initially a warning would be displayed. If the parameter continues to increase out of range the GXD is programmed ${ }^{10}$ to shut itself down.

\section{THE AIRBOX}

The GXD has all of its' components housed inside of a 6.5 "x 6.5 " x 58 ", 0.5 " aluminum airbox which when fully stuffed with components weighs 187 lbs., is electrically grounded at the diagnostic rack in the NIF mezzanine through the cable bundle, but electrically isolated from the NIF chamber. This box design has the advantage of protecting the instrument from data corruption or damage due to potential EMI plus the 1-atm of air inside the box allows for improved cooling for electronics. Additionally, NIF users wanted 
the possibility to operate up to four GXD type instruments in a given DIM for the same experiment.

This forced the spatial dimensions of the airbox to be as small as possible while still modular and user-friendly to maintain.

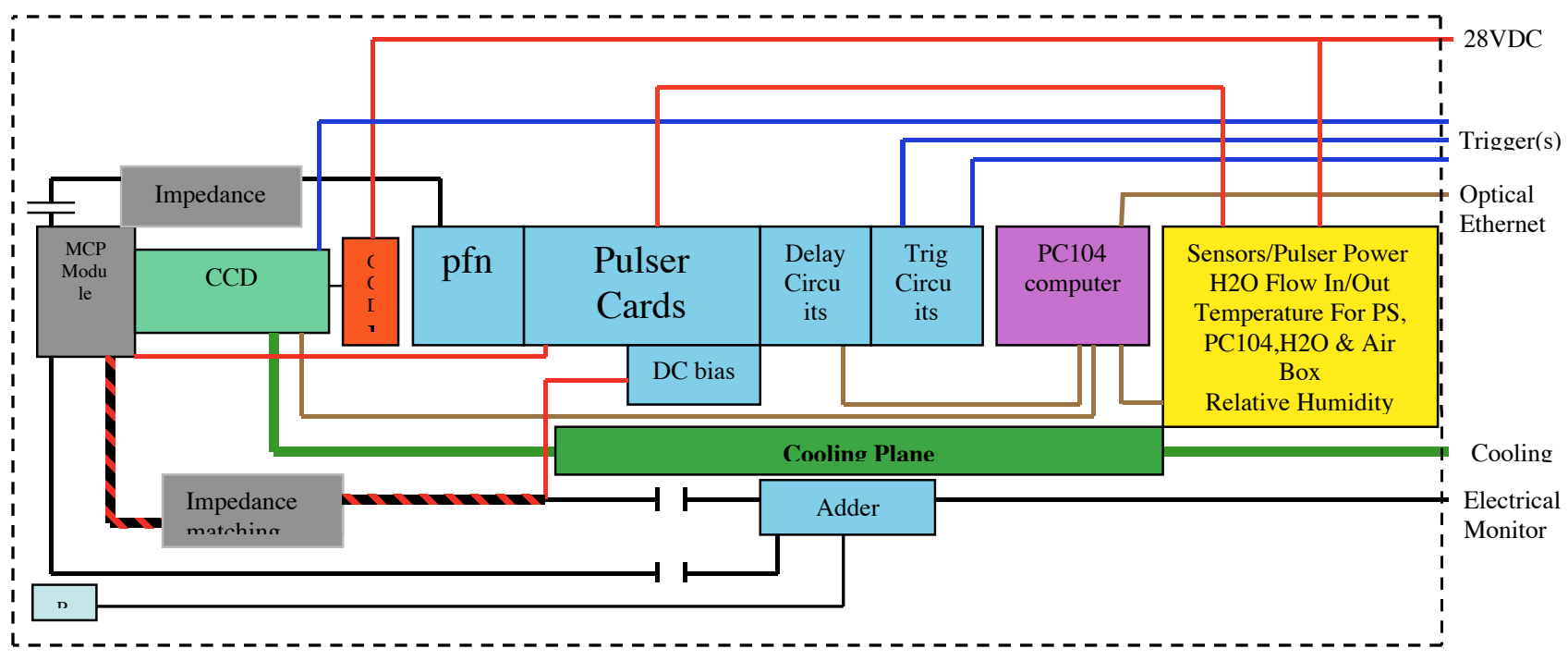

FIG. 2. Block diagram of the Gated X-ray Detector.

\section{COOLING PLANE}

One of the NIF standards and guidelines required the GXD to not dissipate more than 10 watts of heat into the target chamber. NIF engineers were concerned that differential heating of the target positioner could result in target alignment errors. To certify that the GXD would pass this standard, we configured a test-bed to diagnose where and how much heat was generated. The total system heat load for the GXD was experimentally found to be 137.1 watts. The data we acquired from these tests allowed us to optimize a thermal cooling plane that heat-sinked all the heat generating components. With the cooling plane receiving $1.03 \mathrm{~L} / \mathrm{min}$ at $12.7 \mathrm{C}$ all the components came to thermal equilibrium within minutes of power up. Additionally, it was found that when the cooling water was turned off completely the entire system heated up as expected, but took over 120 minutes to reach a critical shut down temperature of $65 \mathrm{C}$. This is an important consideration for GXD operations. If the facility has a cooling water malfunction the GXD can still acquire data up to two hours before the system will shut itself down.

\section{MICROCHANNEL PLATE MODULE AND CCD CAMERA SYSTEM}

The technique used to acquire a time dependent image or spectra is to propagate a high-voltage gate pulse across a microwave transmission stripline deposited on the front surface of a microchannel plate. The short burst of electrons liberated from the Gold photocathode are amplified in the $\mathrm{MCP}^{14}$ and proximity focused onto a p43 phosphor coated fiberoptic faceplate (FOFP). This method of gating is a very mature technique and has been described in detail previously. ${ }^{11}$ 12,13

What makes this system unique is an improved impedance matching scheme, advanced phosphor screen, ability to gate the phosphor voltage and precision assemblies and fixturing.

Past impedance matching systems have either used a direct ohmic mis-match or a shaped transmission line. In the GXD we used a printed circuit, surface mount impedance transformer ${ }^{15}$ going from $50 \Omega$ to the strip impendence of $10 \Omega$. This has the advantage of minimizing reflections back to the gate pulse source and optimizing the energy to the stripline.

On the $6 \mu \mathrm{m}$ pore, Incom ${ }^{16}$ FOFP is a P43 (540 $\mathrm{nm}$ ) phosphor coated to a optimized $1 \mathrm{mg} / \mathrm{cm}^{2}$ thickness and undercoated with a $200 \mathrm{~A}$ indium tin-oxide (ITO) integrated with two Gold tabs for the pulsed phosphor and monitor launch. The cataphoric ${ }^{17}$ deposition technique used on this FOFP resulted in a very uniform, small particle size coating that is very durable and produces greatly improved spatial resolution. ${ }^{18}$ Additionally, we added 3 small unique fiducial marks on the back side of the FOFP so that researchers could easily determine image orientation in their electronic file.

Although not completely unique to these types of diagnostics, pulsing the phosphor screen ${ }^{19}$ for improved spatial resolution (not gating), to date, it is still rare. Pulsing the phosphor screen verses direct current (DC) biasing has the advantage of increasing the electric field between the MCP and FOFP, minimizing 
the transverse energy of the electrons, while avoiding electrical breakdown between the two surfaces. The optimum pulse width of $10 \mu$ s was experimentally determined $^{20}$ to be the point well before breakdown between the MCP and FOFP surfaces but still allowed the voltage to be as high as $5 \mathrm{kV}$ or at $0.5 \mathrm{~mm}$ plate separation an electric field of $1 \times 10^{7} \mathrm{~V} / \mathrm{m}$.

Precision assembly of the MCP module components were put together in a class 100 clean room. The critical components setting the gap between the MCP and FOFP were machined to 0.001 " tolerance or better and electro-polished and cleaned in a ultrasonic bath before being staged in the assembly clean room. Custom designed fixtures were constructed to allow meticulousness control of each component and placement was measured using a laser based proximity sensor. Post assembly, the MCP module is stored in a positive pressure Nitrogen dry box to prevent contamination to moisture.

To capture an image electronically, a liquid cooled, Spectral Instruments SI-1000 CCD camera ${ }^{22,23}$ is fiberoptically coupled to the back of the MCP module. The CCD is held in place using 3 light-weight springs allowing it to "float" and auto-locate on the back of the FOFP. The 16 bit CCD camera is fitted with a $4 \mathrm{k} \times 4 \mathrm{k}$, Kodak KAF16801, class 1 chip that has dimensions of $36.88 \mathrm{~mm}$ square.

\section{GATING ELECTRONICS}

The gating electronics package was custom built by Kentech Instruments LTD $^{24}$ to operate in air, from specifications developed by LANL and LLNL. The electronics are completely computer controlled by interfacing with a standard RS232 connection. Kentech was able to miniaturize the package relative to earlier designs, down to $491 \mathrm{~mm} \times 114 \mathrm{~mm} \times 114 \mathrm{~mm}$. The electronics package includes 4 gating modules that have an output of $\sim 3 \mathrm{kV}$ each with an electrical gate width of 0.2 to 1 ns using changeable pulse forming modules (PFM). These pulses can be delayed up to $44 \mathrm{~ns}$ each in 25 ps steps. Also included are 4 separate DC bias power supplies for independently modifying the gain on each stripline plus DC and pulsed power supplies for the phosphor and photo conductive detector (PCD). Finally, there is a multiplexed monitor circuit that combines the output of the pulsers, pulsed phosphor and PCD that is feed to a high bandwidth oscilloscope.

\section{CONTROL SOFTWARE}

Most of the electrical functions are computer controlled to allow quick adjustment to sensitivity between shots and provide remote monitoring of voltages and currents. The GXD incorporates electronic read-out and controls wherever possible and an automated system for remote setting changes, and the diagnostic is compatible with a 2-hour shot cycle, can store 400 megabytes of data per shot, and is capable of collecting classified data. The GXD Diagnostic Computer (DC) code was developed using LabVIEW 7.0. All communications between the GXD DC code and other hardware and computers occur via built in LabVIEW TCP/IP functions.

\section{SUMMARY}

With the recent completion of the GXD the next phase is to characterize the performance. The initial measurements have been made using bench top fast scopes, the LANL Trident laser facility and the Livermore Bechtel-Nevada calibration facility. Preliminary results, that will be presented in the future, demonstrate very good repeatable uniformity between imaging strips, improved spatial resolution and no detectable impendence reflections.

\section{ACKNOWLEDGEMENTS}

The authors wish to thank the LANL P-24

Diagnostic and Systems Engineering Team and the LANL Trident laser operations crew for help in characterizing the GXD system. This work was performed under the auspices of the U.S. Department of Energy by Los Alamos National Laboratory under contract No. W-7405-Eng-36.

${ }^{1}$ J.D. Kilkenny, Lasers Part. Beams 9, 49 (1991)

${ }^{2}$ C. J. Keane, B. A. Hammel, A. L. Osterheld, and D. R. Kania, Phys. Rev. Lett. 72, 3029-3032 (1994)

${ }^{3}$ G. T. Schappert et al., Bull. Am. Phys. Soc. 41, 1525 (1996)

${ }^{4}$ S.W Haan, Physics of Fluids B 3, (8) August 1991,

${ }^{5}$ R.E Olsen, R.J. Leeper, A. Nobile, J.A. Oertel, Phys. Rev. Lett. 91, 235002 (2003)

${ }^{6}$ T.J. Murphy et. A1., RSI, Volume 72, Number 1, 773, (2001)

${ }^{7}$ D. Kalantar, UCRL-ID-135801 (1999)

${ }^{8}$ J. Oertel, RSI Volume 70, Number 1, 803 (1999)

${ }^{9} \mathrm{~J}$. Holder, To be published. (2007)

${ }^{10}$ Software created by Neal Pederson of VI Controls

Systems Ltd., www.vicontrols.com

${ }^{11}$ O.L. Landen, P. Bell, J. Oertel, J. Satarano, D.

Bradley, SPIE, Vol 2002-1, 2, (1993)

12 J. A. Oertel, L. Schrank T. Archuleta. RSI, Vol 72, No.1, 701 (2001)

${ }^{13}$ P. Bell, J. kilkenny, O. Landen, D. Ress, J. Wiedwald, D. Bradley, J. Oertel, R. Watt, SPIE, Vil. 109, 145-156 (1992)

14 http://www.burle.com

15 "Transmission Line Design Handbook", by Brian C. Wadell, 1991 Artech House, Inc. 685 Canton St,

Norwood, MA 02062, P. 29

$16 \mathrm{http}: / /$ www.incomusa.com

${ }^{17} \mathrm{http}: / /$ lexelimaging.com

${ }^{18}$ To be published, P. Walsh et. al. RSI (2007) 
19 C. Pauli et.al. RSI, Vol. 71, Number 3, 1286, March (2000)

${ }^{20}$ Research commissioned by LLNL to University of Florida, CREOL, 1993

${ }^{21} \mathrm{http}: / /$ world.keyence.com/index.html

${ }^{22} \mathrm{http}: / /$ www.specinst.com

${ }^{23}$ J. Kimbrough et.al. RSI, Vol.72, No. 10, 4060 (2002)

${ }^{24} \mathrm{http}$ ///home.btconnect.com/KENTECH/index.html 